\title{
The Importance of Contextual Understanding in Solving the Last Mile Problem
}

\author{
Craig KUZIEMSKY ${ }^{\mathrm{a}, 1}$ Christian NØHR ${ }^{\mathrm{b}}$, Romaric MARCILLY ${ }^{\mathrm{c}}$, \\ Linda DUSSELJEE-PEUTE ${ }^{\mathrm{d}}$, Xinxin ZHU ${ }^{\mathrm{e}}$, and Peter L. ELKIN ${ }^{\mathrm{f}}$ \\ ${ }^{a}$ MacEwan University, Edmonton, Alberta, Canada \\ ${ }^{\mathrm{b}}$ Danish Centre for Health Informatics, Aalborg University, Denmark \\ ${ }^{\mathrm{c}}$ Univ. Lille, CHU Lille, ULR 2694 - METRICS: Évaluation des technologies de santé \\ et des pratiques médicales, F-59000 Lille, France \\ d Department Medical Informatics, University of Amsterdam, Amsterdam University \\ Medical Center, Amsterdam, the Netherlands

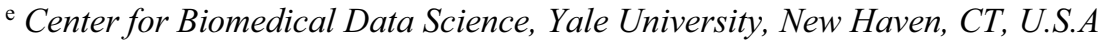 \\ ${ }^{\mathrm{f}}$ Department of Biomedical Informatics, University at Buffalo, U.S.A.
}

\begin{abstract}
The COVID-19 pandemic has disrupted many global industries and shifted the digital health landscape by stimulating and accelerating the delivery of digital care. It has emphasized the need for a system level informatics implementation that supports the healthcare management of populations at a macro level while also providing the necessary support for front line care delivery at a micro level. From data dashboard to Telemedicine, this crisis has necessitated the need for health informatics transformation that can bridge time and space to provide timely care. However, heath transformation cannot solely rely on Health Information Technology (HIT) for progress, but rather success must be an outcome of system design focus on the contextual complexity of the health system where HIT is used. This conference highlights the important roles context plays for health informatics in global pandemics and aims to answer critical questions in four main areas: 1) health information management in the covid-19 context, 2) implementation of new practices and technologies in healthcare, 3) sociotechnical analysis of task performance and workload in healthcare, and 4) innovations in design and evaluation methods of health technologies. We deem this as a call to action to understand the importance of context while solving the last mile problem in delivering the informatics solutions that are needed to support our public health response.
\end{abstract}

Keywords. Pandemic, context, health transformation, health IT

\section{Introduction}

The COVID-19 pandemic shifted the digital health landscape by stimulating the delivery of digital care. When doctors' offices and other care delivery services were forced to close or greatly reduce in person service delivery, digital care delivery became an essential part of the healthcare delivery system $[1,2]$.

\footnotetext{
${ }^{1}$ Corresponding Author, Dr. Craig Kuziemsky, kuziemskyc@macewan.ca
} 
COVID-19 has resulted in almost 4.5 million deaths worldwide. In the USA alone, as of June $17^{\text {th }}, 2021$, over 600,000 people have died from COVID-19 - a rate roughly ten times greater than seasonal influenza deaths [3, 4]. Over 33 million have contracted the infection and a substantial portion of those suffered persisting post-acute symptoms [5]. While COVID-19 case rates and death rates are declining in the US, they are accelerating in other countries such as India. There is a pressing world-wide need for effective therapies [6,7]. Most who die from the COVID-19 suffer from acute respiratory distress syndrome (ARDS) and respiratory insufficiency related to the associated cytokine storm [8].

COVID-19 has focused the importance of informatics driven support for pandemic response. From Telemedicine to COVID 19 research databases the field has risen to the occasion [9, 10]. The pandemic impacted medical education and here Biomedical Informatics rose to the occasion and developed online learning platforms using participatory design to engage our students form home $[11,12]$. Using these techniques our field has stepped up and led biomedicine in weathering the challenge of the pandemic.

The early results on digital care delivery to support global responses to COVID-19 have been mixed. One on hand informatics-based solutions played a fundamental role in the pandemic response that achieved several successes. At a macro level informatics driven solutions were the basis for disease surveillance, contact tracing and vaccine uptake tracking [13-15]. At a micro level informatics solutions enabled patients to access healthcare services through virtual care delivery and telehealth [2, 16]. However, challenges were also identified across the informatics spectrum including a lack of informatics infrastructure or frameworks, data standard or harmonization issues, lack of a skilled informatics workforce, and ethical issues [17]. The COVID-19 driven increase in virtual care delivery raised questions about the design and delivery of virtual care delivery, including patient-provider communication [18].

The informatics response to COVID-19 has reaffirmed one of the foundational problems in medical informatics - "the last mile problem". This problem refers to the difficulty in implementing and integrating informatics models, tools, and approaches into actual care delivery settings so that meaningful outcomes are achieved. The last mile problem is very much central to health system transformation initiatives. Informatics models, tools, or theories alone will not transform healthcare delivery but rather they must lead to meaningful impact at social, policy, economic, cultural, and other levels.

One of the most talked about phenomena in medical informatics are unintended consequences (UICs), which refers to the outcomes that occur but that were not anticipated when implementing health information technology (HIT) [19, 20]. UICs occur because while HIT is a crucial part of healthcare delivery it is only one part of the system of systems that defines healthcare delivery. HIT's interaction with other system components is what leads to UICs [21]. Informatics solutions for COVID-19 have led to UICs including provider fatigue and burnout and inequality in access to digital care delivery $[17,22]$.

A key contributor to the last mile problem is a lack of understanding of the contextual system where HIT is implemented and used. HIT are not generic tools and cannot be implemented without due consideration of the contextual environment where it will be used. We cannot just copy and paste a successful HIT application from one setting to another while expecting success to amplify accordingly. Global health systems can have differences in how healthcare services are funded, delivered, and evaluated. To that end, contextual understanding must be part of any informatics-based implementation. 


\section{Context Sensitive Health Informatics}

The Context Sensitive Health Informatics (CSHI) Conference began in 2013 to provide an arena for presenting research on the contextual nature of HIT implementation. CSHI embraces the messy nature of healthcare delivery and the diversity of settings where HIT is implemented and used. CSHI strives to contribute methods and scientific evidence to advance our understanding of the role context plays in HIT implementation.

Context becomes important when we distinguish automation and transformation. The former is about using technology to digitize existing processes or services whereas the latter is using HIT to achieve a fundamental change to the value statement and means by which services are delivered [23, 24]. This difference is important as health innovation projects can include tasks such as workflow and processes redesign, which requires a greater degree of contextual understanding. HIT should not merely automate existing tasks but rather should help healthcare delivery reach new heights. However, we cannot do that by solely focusing on technology as a driver of health innovation as that may emphasize technology at the expense of contextual considerations such as people, process, and organizational and social considerations.

A key aspect of studying context is recognizing that HIT is implemented and used in a complex system of systems that represent clinical, organizational, technical and policy aspects of healthcare delivery [21]. System context is not about any one system component on its own but rather tries to understand the interactions that occur across system components. Failure to account for how system components influence and reshape context through interactions with each other is a significant barrier to solving the last mile problem. It is therefore necessary to use innovative methods to consider all these components during the design, evaluation and implementation of HIT.

The CSHI 2021 conference highlights the important role context plays for health informatics in global pandemics and aims to answer critical questions in four main areas: 1) health information management in the covid-19 context, 2) implementation of new practices and technologies in healthcare, 3) sociotechnical analysis of task performance and workload in healthcare, and 4) innovations in design and evaluation methods of health technologies. CSHI 2021 provides scientific insight on informatics support for pandemic management and continues the work started at CSHI 2013 (Copenhagen, Denmark), CSHI 2015 (Curitiba, Brazil), CSHI 2017 (Hong Kong, People's Republic of China) and CSHI 2019 (Lille, France).

\section{The Role of Informatics in Global Pandemics}

The COVID-19 pandemic has highlighted the important role that context plays in HIT implementation. Contextual considerations of the role of informatics in managing global pandemics includes:

1. The importance of equity cannot be understated. While informatics solutions such as virtual care enabled patients to access healthcare services during lockdowns it often resulted in uneven access to services. The digital divide was an ongoing problem during informatics responses to COVID-19. We need to ensure that informatics solutions enable better access to care delivery for everyone rather resulting in equity issues for certain patient populations. 
2. Not all healthcare services transitioned effectively to digital delivery. Complex physical assessments can be challenging to do digitally. We cannot simply use technology as a complete replacement for tasks that are best delivered via human touch. The COVID-19 mediated transition to digital care delivery resulted in a lot of trial and error to determine what worked best in certain contextual situations. Going forward we need evidence as to which clinical processes transition well to digital delivery and which ones should remain faceto-face.

3. Public and clinical informatics systems must be better coordinated. COVID-19 illustrated that gaps exist in the connection between micro level clinical care and public health at the macro level. These gaps impacted pandemic management by making contact tracing and disease surveillance difficult. A well-connected health system is the underpinning of an efficient pandemic response.

4. Health transformation must focus on meaningful outcomes of care delivery. While technology may automate a specific process, healthcare transformation occurs at the system level and we must account for all system components (e.g., human resource, organizational, financial, political, technological) during health transformation efforts.

5. Automation happens in the moment. Transformation happens over time. Digital transformation of processes such as decision support or patient-provider communication is a formative task that takes place over a continuum. Health transformation cannot be implemented or evaluated as a one-off event but rather must be done under principles of a learning health system [25].

6. Understanding context requires meaningful engagement with all users of HIT. This includes providers, patients, policy makers, decision makers and any other end users.

\section{Future Directions Towards Solving the "Last Mile Problem"}

The COVID-19 pandemic reaffirmed that healthcare delivery is complex and that contextual variation contributes to the complexity. Healthcare is delivered in complex systems and until we understand these systems and the role that context plays in these systems; we will continue to have problems with health transformation. Heath transformation cannot rely on HIT to guarantee progress or success, but rather success must be an outcome of system design. However, system design is challenging due to the need to purposefully design for the contextual environments where healthcare services are delivered. We must resist the urge to focus on technology and instead focus on the contextual complexity of the health system where HIT is used. HIT failures are rarely due to bad technology per se but rather are due to a lack of contextual alignment with the setting where the HIT is used. Informatics projects or research may start starts on the fast track in solving the first mile problem only to fail to deliver in the last mile. A lack of attention to context is often the barrier that prevents HIT from solving the last mile problem. Do not underestimate the role that context plays in HIT implementation as context is always a factor.

Without due attention to context in how we design, implement, and evaluate HIT we will continue to struggle with the last mile problem. These challenges also present opportunities for various stakeholders such as vendors and public health decision makers 
to foster and support innovations and health system transformation for a better health system for everyone. Future research is needed to better understand the human factors and sociotechnical issues related to how to use HIT to meaningfully connect individuals with our public health authorities in times of crisis such as a global pandemic. The CSHI is committed to reporting new developments and progress in future medical informatics conferences.

\section{References}

[1] J. Wosik, M. Fudim, B. Cameron, Z.F. Gellad, A. Cho, D. Phinney et al., Telehealth transformation: COVID-19 and the rise of virtual care, J Am Med Inform Assoc 27 (2020), 957-62.

[2] S.T. Liaw, C. Kuziemsky, R. Schreiber, J. Jonnagaddala, H. Liyanage, A. Chittalia et al., Primary Care Informatics Response to Covid-19 Pandemic: Adaptation, Progress, and Lessons from Four Countries with High ICT Development, Yearb Med Inform 30 (2021), 44-55.

[3] COVID-19 Map - Johns Hopkins Coronavirus Resource Center. https://coronavirus.jhu.edu/map.html

[4] Past Seasons Estimated Influenza Disease Burden | CDC. https://www.cdc.gov/flu/about/burden/pastseasons.html.

[5] E.M. Amenta, et al., Postacute COVID-19: An Overview and Approach to Classification. Open Forum Infect Dis 7 (2020).

[6] V.V. Kouznetsov, COVID-19 treatment: Much research and testing, but far, few magic bullets against SARS-CoV-2 coronavirus, Eur J Med Chem 203 (2020).

[7] HHS News Division. Biden Administration to Invest \$3 Billion from American Rescue Plan as Part of COVID-19 Antiviral Development $\quad$ Strategy. $\quad$ HHS.gov https://www.hhs.gov/about/news/2021/06/17/biden-administration-invest-3-billion-american-rescueplan-as-part-covid-19-antiviral-development-strategy.html

[8] S. M. Abdin, S.M. Elgendy, S.K. Alyammahi, D.W. Alhamad, and H. A. Omar, Tackling the cytokine storm in COVID-19, challenges and hopes, Life Sci 257 (2020).

[9] T.D. Bennett, R.A. Moffitt, J.G. Hajagos, B. Amor, A. Anand, M. M. Bissell et al., National COVID Cohort Collaborative (N3C) Consortium. Clinical Characterization and Prediction of Clinical Severity of SARSCoV-2 Infection Among US Adults Using Data From the US National COVID Cohort Collaborative, JAMA Netw Open 4 (2021): e2116901.

[10] M.A. Haendel, C.G. Chute, T.D. Bennett, D.A. Eichmann, J. Guinney, W.A. Kibbe et al., The National COVID Cohort Collaborative (N3C): Rationale, design, infrastructure, and deployment, J Am Med Inform Assoc 28 (2021), 427-443.

[11] G. Franklin, C. Martin, M. Ruszaj, M. Matin, A. Kataria, J. Hu et al., How the COVID-19 Pandemic Impacted Medical Education during the Last Year of Medical School: A Class Survey, Life (Basel) 11 (2021), 294.

[12] B. MacKenzie, G. Anaya, J. Hu, A. Brickman, P. L. Elkin, M. Panesar, Defining Data Migration Across Multidisciplinary Ambulatory Clinics Using Participatory Design, Appl Clin Inform 12 (2021), 251-258.

[13] B. Tilahun, K. D. Gashu, Z. A. Mekonnen, B.F. Endehabtu, and D.A. Angaw, Mapping the Role of Digital Health Technologies in Prevention and Control of COVID-19 Pandemic: Review of the Literature, Yearb Med Inform 30 (2021), 26-37.

[14] R.A. Kleinman, C. Merkel. Digital contact tracing for COVID-19. CMAJ 192 (2020): E653-E656.

[15] B. Glampson, J. Brittain, A. Kaura, A. Mulla, L. Mercuri, S. J. Brett et al., Assessing COVID-19 Vaccine Uptake and Effectiveness Through the North West London Vaccination Program: Retrospective Cohort Study, JMIR Public Health Surveill 7 (2021): e30010.

[16] A. Basu, C. Kuziemsky, M. de Araújo Novaes, A. Kleber, F. Sales, N. Al-Shorbaji et al., Telehealth and the COVID-19 Pandemic: International Perspectives and a Health Systems Framework for Telehealth Implementation to Support Critical Response, Yearb Med Inform 30 (2021), 126-133.

[17] M.A. Basit, C.U. Lehmann, R.J. Medford. Managing Pandemics with Health Informatics: Successes and Challenges, Yearb Med Inform 30 (2021), 17-25.

[18] E. Wittenberg, J. V. Goldsmith, C. Chen, M. Prince-Paul, and R. R. Johnson, Opportunities to improve COVID-19 provider communication resources: A systematic review, Patient education and counseling 104 (2021), 438-451.

[19] E. Coiera, J. Ash, and M. Berg, The Unintended Consequences of Health Information Technology Revisited. Yearb Med Inform 1 (2016), 163-169. 
[20] K. Zheng, J. Abraham, L. L. Novak, T. L. Reynolds, A. Gettinge, A Survey of the Literature on Unintended Consequences Associated with Health Information Technology: 2014-2015 Yearb Med Inform 1 (2016), 13-29.

[21] C. Champion, C. Kuziemsky, E. Affleck, G. G. Alvarez, A systems approach for modeling health information complexity, Int J of Inform Manag 49 (2019), 343-54.

[22] A. Ramsetty, C. Adams, Impact of the digital divide in the age of COVID-19, J Am Med Inform Assoc 27 (2020), 1147-1148.

[23] T. Greenhalgh, J. Wherton, C. Papoutsi, J. Lynch, G. Hughes, C. A'Court, et al., Beyond Adoption: A New Framework for Theorizing and Evaluating Nonadoption, Abandonment, and Challenges to the Scale-Up, Spread, and Sustainability of Health and Care Technologies, J Med Internet Res 19 (2017): e367

[24] L. Wessel, A. Baiyere, R. Ologeanu-Taddei, J. Cha, and T. Blegind Jensen, Unpacking the Difference Between Digital Transformation and IT-Enabled Organizational Transformation, $J$ of the Ass for Inform Syst 19 (2021): e367.

[25] C. Friedman, J. Rubin, J. Brown, M. Buntin, M. Corn, L. Etheredge, et al., Toward a science of learning systems: a research agenda for the high-functioning Learning Health System, J Am Med Inform Assoc 22 (2015), 43-50. 